\title{
Geometric Methods in Multiparticle Quantum Systems
}

\author{
Barry Simon* \\ Department of Physics, Yeshiva University, New York, NY 10033, USA
}

\begin{abstract}
Technically simple proofs are given of the HVZ theorem on the bottom of the essential spectrum of multiparticle systems and of Combes' result on completeness below the lowest three body threshold. The first proof is a variant of a proof of Enss and a decendent of Zhislin's original proof. Finally, we apply our methods to the bound state spectrum.
\end{abstract}

\section{$\S 1$. Introduction}

This is the second of a series of papers that attempts to develop the basic spectral and scattering properties of multiparticle nonrelativistic Schrödinger operators without the use of resolvent equations. The first paper in the series, written jointly with P. Deift, [15], showed how to reduce the completeness of the scattering theory to the existence of certain time-dependent operators (which remain to be controlled). In that paper an important element was the idea of using the geometry of configuration space to separate channels. This idea is basic to the present paper; indeed it is the central character in the drama with various technical results playing merely supporting roles.

My paper with Deift was not the first one to suggest the use of time-dependent methods in studying the completeness of multichannel multiparticle systems. In 1967, J. M. Combes [9] published an extremely deep paper on completeness of $N$ particle systems below the lowest energy necessary for breakup into three or more clusters. This paper has been largely ignored, probably in part because it is technically somewhat complex. My original motivation in the present work was a desire to use the insights of [15] to find a simpler proof of Combes' result. I expected (correctly so) that the idea of Pearson [29] of systematically exploiting the "two Hilbert space" operator

$$
\Omega^{ \pm}(A, B ; J)=\underset{t \rightarrow \mp \infty}{S-\lim _{t \rightarrow \infty}} e^{i A t} J e^{-i B t} E_{\mathrm{ac}}(B)
$$

* On leave from Princeton University. Research support in part by U.S. National Science Foundation under grants MPS-75-11864 and MPS-75-20638 
would be important. Combes' result is proven in $\S 3$. In finding this proof, I had to overcome a technical problem [Step (1) in the scheme of $\S 3$ ] whose solution led to a remarkably simple proof of the HVZ theorem, i.e. the result of Hunziker [22], Van Winter [46] and Zhislin [49] on the location of the bottom of the essential spectrum. This proof is clearly a decendent of the original proof of Zhislin, a method systematically developed by Jörgens [24] and Jörgens-Weidmann [25], but I feel it is technically more transparent. I also remark that the "easy" half of the Hunziker's proof is "geometric". After the completion of this phase of my work, I learned from J. Fröhlich of a beautiful preprint of V. Enss [18] who also presents a decendent of the Zhislin proof which is technically quite transparent; in fact, the proof in $\S 2$ should be regarded as a relatively minor variant of his. Given Enss' work, I have included a proof of the HVZ theorem for two reasons: first given what I need to prove for $\S 3$ and $\S 4$, it is essentially a remark that the HVZ theorem has been proven. Secondly, I wish to present what seems to be the "proper" form of the theorem with symmetries (Theorem 2.6) and to emphasize an elementary approximation result (Proposition 2.2) whose import for the HVZ problem (Theorem 2.3) appears to have escaped notice.

As already stated, $\S 3$ presents a proof of Combes' result on completeness of the scattering below the lowest three body threshold for sufficiently short range potentials. I emphasize that most of my steps are essentially in Combes and that, in particular steps (2) and (4) are explicitly in his paper. What I have succeeded in doing is eliminating a certain amount of excess baggage from his proof.

In $\S 4$, we turn to the question of when multiparticle systems have finitely many bound states below the essential spectrum. The bulk of the results have been obtained already using resolvent equation by Yafeev [47,48] and Sigal [36], but only with some considerable effort. My approach has considerable overlap with a method Combes outlined to me in a letter [11] in the fall of 1972; for some reason, he has chosen to never publish any details. A theorem of the genre of Theorem 4.1 occurs in his work but with more technical preliminaries. In addition, Thirring [45] has not unrelated methods.

I should like to emphasize that the fundamental ideas used below are already in the work of Zhislin and Combes and that Enss' beautiful paper provides a conceptual similar and technical simple proof of the HVZ theorem. However, it seems to me that there is some point in seeing the sweep of results presented here obtained by geometric methods and without the pernicious influence of resolvent equations. Not that these equations do not have their usefulness but it seems to me that they have overly dominated our thinking. I will regard this paper as a success if it helps strike a balance in the reader's mind; even better, I would hope to convey the excitement of the geometric ideas.

The basic idea in the whole paper is that in regions of configuration space where clusters $C_{1}$ and $C_{2}$ are far apart, the total Hamiltonian $H$ of the system should "look like" the Hamiltonian $H_{\alpha}$ of the system with the potentials between $C_{1}$ and $C_{2}$ removed. It will be useful to have a very strong notion of "look like" by proving that functions of $H$ and $H_{\alpha}$ are close to one another in operator norm. The following elementary result is thus at the heart of the technical details: 
Theorem 1.1. Let $H_{1}$ and $H_{2}$ be two self-adjoint operators on a Hilbert space, $\mathscr{H}$, both bounded from below. Let $A_{n}$ be a sequence of bounded operators with $\sup \left\|A_{n}\right\|<\infty$. Suppose that

$$
\lim _{n \rightarrow \infty}\left\|A_{n}\left[\left(H_{1}-z\right)^{-1}-\left(H_{2}-z\right)^{-1}\right]\right\|=0
$$

for $z$ in some open set of $(-\infty, c) \subset R$ where $c=\min \left[\inf \sigma\left(H_{i}\right)\right]$. Then

$$
\lim _{n \rightarrow \infty}\left\|A_{n}\left(f\left(H_{1}\right)-f\left(H_{2}\right)\right)\right\|=0
$$

for any bounded continuous function on $R$ going to zero at $\infty$.

Proof. This is a simple modification of the proof in [30] of the continuity of the functional calculus (Theorem VIII.20 of [30]). The operators on the left side of (1.2) are uniformly bounded in $n$ and $z$ as $z$ runs though compact subsets of $C \backslash[c, \infty)$, so by the Vitali convergence theorem, (1.2) holds for all such $z$ and the convergence is uniform on compact subsets of $C \backslash[c, \infty)$. In particular, by Cauchy's formula the derivatives converge in norm so that

$$
\left\|A_{n}\left[\left(H_{1}-c+1\right)^{-m}-\left(H_{2}-c+1\right)^{-m}\right]\right\| \rightarrow 0
$$

for any $m$. By the Stone-Weierstrass theorem, given $\varepsilon$, we can find a polynomial $P_{\varepsilon}(X)$ so that

$$
\sup _{x \geqq c}\left|f(x)-P_{\varepsilon}\left((x-c+1)^{-1}\right)\right| \leqq \varepsilon
$$

(1.3) follows from (1.5) and (1.4).

It is a pleasure to thank J. Avron, P. Deift, and I. Herbst for valuable comments and encouragement, E. Lieb for a valuable remark, J. Fröhlich for telling me of the work of V. Enss, and P. Deift and R. Kadison for valuable correspondence concerning the material in Appendix A.

\section{§2. The HVZ Theorem}

Consider the Hamilton, $H$, of a system of $N m$-dimensional particles with center of mass motion removed. Let $\alpha=1, \ldots, K \equiv 2^{N-1}-1$ be a labelling of all the ways of breaking $\{1, \ldots, N\}$ into two non-empty disjoint subsets $C_{1}^{(\alpha)}, C_{2}^{(\alpha)}$. Let $H_{\alpha}$ be $H-I_{\alpha}$ where $I_{\alpha}$ is the sum of all interactions involving some particles from each cluster. The HVZ theorem is the assertion that

$$
\sigma_{\text {ess }}(H)=[\Sigma, \infty) ; \Sigma=\inf _{\alpha}\left(\inf \sigma\left(H_{\alpha}\right)\right)
$$

under suitable hypotheses. (See [30] for definition of essential spectrum, etc.) Here, we wish to consider only the case of local pair potentials although it is clear that the method extends to multiparticle local potentials and a variety of nonlocal potentials, presumably as general as those in [25]. At the conclusion of the section, we make a few remarks about including magnetic fields.

We begin with two elementary functional analytic results which we first learned about from a paper of Glimm and Jaffe [20]. Although they appear essentially as Theorems XIII.77 and XIII.78 of [33], we give their simple proofs: 
Proposition 2.1. Let $H$ be a self-adjoint operator. An open subset $\Omega \subset R$ is disjoint from $\sigma_{\mathrm{ess}}(H)$ if and only if $f(H)$ is compact for every continuous function $f$ of compact support with supp $f \subset \Omega$.

Proof. Suppose that $\sigma(H) \cap \Omega \subset \sigma_{\text {disc }}(H)$. Then, for any $K \subset \Omega$, the spectral projection $E_{K}(H)$ is finite rank and so compact. Let $\operatorname{supp} f \equiv K$ be compact and in $\Omega$. Then $|f(H)|^{2} \leqq\|f\|_{\infty}^{2} E_{K}(H)$ so $f(H)$ is compact. Conversely, if every such $f(H)$ is compact and $K \subset \Omega$ is compact, find such an $f$ with $\chi_{K} \leqq \mathrm{f}$ where $\chi_{K}$ is the characteristic function of $K$. Then $E_{K}(H) \leqq f(H)$, so $E_{K}(H)$ is compact and so finite rank.

This proposition is the replacement of Weyl-type criteria used in earlier geometric proofs of the HVZ theorem [29, 25, 18].

Proposition 2.2. Let $H_{n}, H$ be self-adjoint operators with $\left\|\left(H_{n}-z\right)^{-1}-(H-z)^{-1}\right\| \rightarrow 0$ for each fixed $z \in C \backslash R$ as $n \rightarrow \infty$. Suppose $\sigma_{\text {ess }}\left(H_{n}\right)=\left[\Sigma_{n}, \infty\right)$. Then $\Sigma_{n} \rightarrow \Sigma$ (which may be $\pm \infty)$ as $n \rightarrow \infty$ and $\sigma_{\text {ess }}(H)=[\Sigma, \infty)$.

Proof. Pass to a subsequence so that $\Sigma_{n}$ converges to some $\Sigma$. If we prove that $\sigma_{\text {ess }}(H)$ $=[\Sigma, \infty)$ we will have that $\Sigma$ is independent of subsequence so that the original limit converges. Let $a>\Sigma$. If $a \notin \sigma(H)$, then, for all large enough $n, a \notin \sigma\left(H_{n}\right)$ (see [30], Theorem VIII.23) which is impossible since $a>\Sigma_{n}$ for $n$ large enough. Thus $[\Sigma, \infty) \subset \sigma(H)$. On the other hand, let $a<b<\Sigma$. Let $f$ be a continuous function with support in $(a, b)$. By the continuity of the functional calculus (see [30], Theorem VIII.20), $f\left(H_{n}\right) \rightarrow f(H)$ in norm. Since $b<\Sigma_{n}$ for $n$ large, $f\left(H_{n}\right)$ is compact for $n$ large by Proposition 2.1, $f(H)$ is compact, so again by Proposition 2.1, $(-\infty, \Sigma) \subset \sigma_{\mathrm{disc}}(H)$.

Theorem 2.3. If the HVZ theorem, (2.1), is proven for two body potentials in $C_{0}^{\infty}\left(R^{m}\right)$, it holds for two body potentials in $L^{p}\left(R^{m}\right)+L_{\varepsilon}^{\infty}\left(R^{m}\right)$ with $p=1$ if $m=1, p>1$ if $m=2$ and $p=m / 2$ for $m \geqq 3$.

Proof. Note the following abstract result (see Theorem VIII.25 of [30]): If $H_{0}$ is a fixed positive self-adjoint operation, if $V_{n}$ are quadratic forms with $Q\left(H_{0}\right) \subset Q(V)$ and

$$
\pm\left(\varphi, V_{n} \varphi\right) \leqq \alpha\left(\varphi,\left(H_{0}+C\right) \varphi\right)
$$

for some fixed $\alpha<1$ and $c>0$ and if

$$
\pm\left(\varphi,\left(V-V_{n}\right) \varphi\right) \leqq \alpha_{n}\left(\varphi,\left(H_{0}+1\right) \varphi\right)
$$

with $\alpha_{n} \rightarrow 0$ as $n \rightarrow \infty$, then $\left(H_{0}+V_{n}-z\right)^{-1} \rightarrow\left(H_{0}+V-z\right)^{-1}$ for all $z \in C \backslash R$. From this fact, the standard Sobolev and Strichartz type estimates (see e.g. Theorem X.21 of [31]), the density of $C_{0}^{\infty}$ in $L^{p}+L_{\varepsilon}^{\infty}$ and Proposition 2.2, we conclude the proof.

The point of this result and its generalization to other cases where Proposition 2.2 is applicable (e.g. the situation of [25]) is that extensions of the HVZ theorem are easy. For example, Simon [37] need not have developed the factorized Weinberg-van Winter machinery for his proof of the HVZ theorem with Rollnik potentials and Jörgens-Weidmann were being overly critical of their work when they said ([25], p. 5): "Our results contain all earlier results besides the result given by Simon; this is actually not comparable with our result."

We will therefore give our proof below for potentials in $C_{0}^{\infty}$, although as we remark and as we will need later, the proof works for general potentials. For any 
$x=\left(x_{1}, \ldots, x_{N}\right) \in R^{N m}$, define

$$
\begin{aligned}
|x| & =\left(\sum_{i<j}\left|x_{i}-x_{j}\right|^{2}\right)^{1 / 2} \\
|x|_{\alpha} & =\min \left\{\mid x_{i}-x_{j} \| i \in C_{\alpha}^{(1)}, j \in C_{\alpha}^{(2)}\right\} .
\end{aligned}
$$

Both $|x|$ and $|x|_{\alpha}$ are independent of the center of mass coordinate, so we can define them on $R^{(N-1) m}$ after removing the center of mass motion. The following geometric fact is critical:

Lemma 2.4. For any $x$, there is some $\alpha \in\{1, \ldots, K\}$ so that $|x|_{\alpha} \geqq d_{N}|x|$ where $d_{N}$ $=\sqrt{2} N^{-1 / 2}(N-1)^{-3 / 2}$.

Proof. Given, $x$ pick $i, j$ so that $\left|x_{i}-x_{j}\right|$ is maximal. Then $R \equiv\left|x_{i}-x_{j}\right| \geqq \sqrt{2}|x| N^{-1 / 2}$ $(N-1)^{-1 / 2}$. Pick coordinates in $R^{n}$ so that $x_{j}=0$ and $x_{i}=(R, 0, \ldots, 0)$. Consider the $(N-1)$ regions:

$$
R_{l}=\left\{x \mid R(N-1)^{-1}(l-1)<x^{(1)}<R(N-1)^{-1} l\right\}
$$

$l=1, \ldots, N-1$ where $x^{(1)}$ is the 1 st coordinate of $x$. Since there are only $N-2$ points other than $i$ and $j$ some $R_{l}$ must contain no points. Pick $\alpha$ so that $C_{\alpha}^{(1)}$ contains those particles $k$, with $x_{k}^{(1)} \leqq R(N-1)^{-1}(l-1)$ and $C_{\alpha}^{(2)}$ those with $x_{k}^{(1)} \geqq R(N-1)^{-1} l$. Clearly $|x|_{\alpha} \geqq R(N-1)^{-1}$.

Now pick functions $j_{\alpha}$ on $R^{(N-1) m}$ which are $C^{\infty}$ away from 0 , homogeneous of degree zero [i.e. $\left.j_{\alpha}(x)=j_{\alpha}(x /|x|)\right]$ so that $0 \leqq j_{\alpha} \leqq 1$ and

(i) $j_{\alpha}(x)=0$ if $|x|_{\alpha} \leqq \frac{1}{2} d_{N}|x|$

(ii) $\sum_{\alpha=1}^{K} j_{\alpha}(x)=1$.

By the lemma, such functions exist. Notice, that by the homogeneity, $\nabla j_{\alpha}=0(1 /|x|)$ at infinity.

Warning. While they play a somewhat similar role, the $j_{\alpha}$ are different from the $J_{\alpha}$ of [15] not only quantitatively but qualitatively: $j_{\alpha}$ lives in the region where the clusters $C_{\alpha}^{(1)}$ and $C_{\alpha}^{(2)}$ are not close to each other while $J_{\alpha}$ lives in the region where, in addition, the particles in each cluster are close to one another.

Pick a fixed function, $\varphi$, in $C_{0}^{\infty}\left(R^{(N-1) n}\right)$ which is 1 if $|x| \leqq 1$ and 0 if $|x| \geqq 2$. Let

$J_{\leqq R}(x)=\varphi(x / R)$

$J_{\geqq R}(x)=1-J_{\leqq R}$.

The equation we will use again and again is the following identity:

$$
f(H)=f(H) J_{\leqq n}+\sum_{\alpha} f\left(H_{\alpha}\right) j_{\alpha} J_{\geqq n}+\sum_{\alpha}\left(f(H)-f\left(H_{\alpha}\right)\right) j_{\alpha} J_{\geqq n} .
$$

Theorem 2.5. The HVZ equality, (2.1), holds when all potentials are in $C_{0}^{\infty}$.

Proof. We will only concern ourselves with the "hard" direction $\sigma(H) \cap(-\infty, \Sigma) \subset \sigma_{\text {disc }}(H)$. One can actually prove the easy direction from an equation like (2.2); We note that Hunziker's proof of the easy direction is also geometric. Since the potentials have compact support, we can find $R_{0}$ so that $V_{i j}(y)=0$ if $y \in R^{n}$ with $|y| \geqq R_{0}$. It follows that $I_{\alpha}(x)=0$ if $|x|_{\alpha} \geqq R_{0}$ so that, by 
construction of $j_{\alpha}, I_{\alpha} j_{\alpha} J_{\geqq n}=0$ if $n \geqq 2 d_{N}^{-1} R_{0}$. For such $n$ :

$$
\begin{aligned}
& {\left[(H-z)^{-1}-\left(H_{\alpha}-z\right)^{-1}\right] j_{\alpha} J_{\geqq n}} \\
& =-\left(H_{\alpha}-z\right)^{-1} I_{\alpha}(H-z)^{-1} j_{\alpha} J_{\geqq n} \\
& =-\left(H_{\alpha}-z\right)^{-1} I_{\alpha}\left[(H-z)^{-1}, j_{\alpha} J_{\geqq n}\right]
\end{aligned}
$$

which goes to zero in norm as $n \rightarrow \infty$ since the commutator $\left[(H-z)^{-1}, j_{\alpha} J_{\geqq n}\right]$ is bounded by $C\left(\left\|J_{\geq n} \nabla j_{\alpha}\right\|_{\infty}+\left\|\nabla J_{\geq n}\right\|\right)=0(1 / n)$. Thus, by Theorem 1.1, the last term in (2.2) goes to zero in norm as $n \rightarrow \infty$ for any $f$ which is continuous with compact support in $(-\infty, \Sigma)$. By definition, of $\Sigma$, for $\operatorname{such} f$, each $f\left(H_{\alpha}\right)=0$ so the second term is zero. Finally

$$
f(H) J_{\leqq n}=[f(H)(H+1)]\left[(H+1)^{-1}\left(H_{0}+1\right)\right]\left[\left(H_{0}+1\right)^{-1} J_{\leqq n}\right]
$$

is compact since the first two factors are bounded and the last is compact. It follows that $f(H)$ is compact, so $\sigma_{\text {disc }}(H) \supset(-\infty, \Sigma)$ by Proposition 2.1.

Now consider the situation where $H$ has a "symmetry", i.e. there is a projection $P$ reducing $H$. Then since $\Sigma \leqq 0, f(0)=0$ so that $f(H P)=P f(H)$. Looking at the analysis of (2.2), we see that the first and third term can be treated as before, so we only need the middle term to be zero. Let $P_{\alpha}^{(0)}$ be the smallest projection reducing $H_{\alpha}$ and containing $P$. Then $P f\left(H_{\alpha}\right)=P P_{\alpha}^{(0)} f\left(H_{\alpha}\right)=P f\left(P_{\alpha}^{(0)} H_{\alpha}\right)$. Thus, we have:

Theorem 2.6. Let $P$ be a projection reducing $H$ and let $P_{\alpha}$ be any projection reducing $H_{\alpha}$ with $\operatorname{Ran} P_{\alpha} \supset \operatorname{Ran} P$. Let $\Sigma_{P}=\min _{\alpha}\left\{\inf \sigma\left(H_{\alpha} P_{\alpha}\right)\right\}$. Then

$$
\sigma(H P) \cap\left(-\infty, \Sigma_{P}\right) \subset \sigma_{\text {disc }}(H P) \text {. }
$$

It will be seen that this theorem contains the "hard" part of all previous "symmetry" theorems [50, 51, 54, 38, 4, 25].

We close this section with four remarks:

(1) The only place we used the fact that the $V$ 's had compact support was in estimating $\left[(H-z)^{-1}-\left(H_{\alpha}-z\right)^{-1}\right]$. It is clearly sufficient that $\left\|\left(H_{\alpha}-z\right)^{-1} I_{\alpha} j_{\alpha} J_{\geqq n}(H-z)^{-1}\right\|$ go to zero as $n \rightarrow \infty$. This will happen if the potentials lie in $L^{p}+L_{\varepsilon}^{\infty}$ where $p$ is as in Theorem 2.3 so that Theorem 2.3 plays no direct rolewe quoted it merely for its "historical" significance. In $\S \S 3$ and 4 , we will use freely the fact that the last term in (2.2) goes to zero as $n \rightarrow \infty$ in this more general setting

(2) The method easily accomodates external magnetic fields which by their nature do not contribute to $I_{\alpha}$. In the estimates, it is useful to have the result of Avron et al. [3] that if $V$ is $-\Delta$-bounded, it is $-\Sigma\left(\partial_{j}-i a_{j}\right)^{2}$-bounded with the same or smaller relative bound for any $a_{j} \in L_{\text {loc }}^{2}$. It follows that the HVZ theorem extends to the case of such $a_{j}$ 's and the precise potentials of Theorem 2.3.

(3) The present method is not only physical transparent but yields some results in a stronger form than does the resolvent equation approach. It's main drawback as presented is its reliance on the self-adjointness of $H$. This makes it unsuitable for certain applications such as that needed in the analysis of dilation analytic potentials $[5,39]$. This defect can probably be remedied.

(4) [This answers a question raised by Hill (private communication)]: The method easily accomodates severe local singularities such as occur with hard cores or Lenard-Jones potentials. The key observation is that in $\left(H_{\alpha}-z\right)^{-1} I_{\alpha}\left[(H-z)^{-1}, j_{\alpha} J_{\geq n}\right]$, the term $\left(H_{\alpha}-z\right)^{-1} I_{\alpha}(H-z)^{-1}$ can be controlled by writing it as $\left(H_{\alpha}-z\right)^{-1}-(\bar{H}-z)^{-1}$. 


\section{§3. Completeness in the Two Body Region}

In the notation of the last section, each breadup, $\alpha$, into two clusters induces a tensor decomposition of $L^{2}\left(\mathbb{R}^{m(N-1)}\right)$ corresponding to coordinates $\left(x_{\alpha}, y_{\alpha}\right)$ where $y_{\alpha} \in \mathbb{R}^{m}$ is the difference of the center of mass of the clusters and $x_{\alpha}=\left(x_{\alpha}^{(1)}, x_{\alpha}^{(2)}\right)$ is "internal" coordinates for $C_{\alpha}^{(1)}, C_{\alpha}^{(2)}$. To distinguish the different discompositions as $\alpha$ varies, we will write operators as $A \otimes_{\alpha} B$. Then

$$
H_{\alpha}=h_{\alpha} \otimes_{\alpha} 1+1 \otimes_{\alpha} T_{\alpha}
$$

where $T_{\alpha}$ is the kinetic energy of the clusters. Let

$$
\begin{aligned}
& \Sigma_{2, \alpha} \equiv \inf \sigma\left(h_{\alpha}\right) ; \quad \Sigma_{2}=\min _{\alpha} \Sigma_{2, \alpha} \\
& \Sigma_{3, \alpha} \equiv \inf \sigma_{\text {ess }}\left(h_{\alpha}\right) ; \quad \Sigma_{3}=\min \Sigma_{3, \alpha} .
\end{aligned}
$$

By the HVZ theorem, $\Sigma_{3}$ is the lowest energy for breakup into three clusters.

It will often happen that $\Sigma_{2}<\Sigma_{3}$ in which case scattering in $\left[\Sigma_{2}, \Sigma_{3}\right]$ will consist of two body rearrangements. In that case, let $p_{\alpha}$ be the projection into all the eigenfunctions of $h_{\alpha}$ with energy $e<\Sigma_{3}$ and $P_{\alpha}=p_{\alpha} \otimes_{\alpha} 1$.

Under fairly general conditions, the wave operators $\Omega_{\alpha}^{ \pm}=\Omega^{ \pm}\left(H, H_{\alpha}\right)$ exist (see Reed-Simon [32] for a general discussion including detailed historical references) and the operators

$$
\hat{\Omega}_{\alpha}^{ \pm} \equiv \Omega^{ \pm}\left(H, H_{\alpha}\right) P_{\alpha}=\Omega^{ \pm}\left(H, H_{\alpha} ; P_{\alpha}\right)
$$

have orthogonal ranges.

Warning. $P_{\alpha}$ and $\hat{\Omega}_{\alpha}^{ \pm}$are slightly different from those in [15] but they are the same in the energy range under consideration.

The following result is essentially due to Combes [9]:

Theorem 3.1. Suppose that each two body potential $V_{i j}$ obeys

$$
\left(1+|y|^{2}\right)^{(1 / 2 m+\varepsilon)} V_{i j}(y) \in L^{p}\left(\mathbb{R}^{m}\right)+L^{\infty}\left(\mathbb{R}^{m}\right)
$$

where $p \geqq 2, p>m / 2$. Then

$$
\operatorname{Ran} \tilde{E} \equiv \operatorname{Ran} E_{\mathrm{ac}}(H) E_{\left(-\infty, \Sigma_{3}\right)}(H) \subset \bigoplus_{\alpha} \operatorname{Ran} \hat{\Omega}_{\alpha}^{ \pm} .
$$

Remarks. 1. (3.1) say essentially that $V(y)=0\left(|y|^{-m-\varepsilon}\right)$ at infinity. This is the wellknown borderline for applicability of the trace class theory [32].

2. In terms of the EBFM wave operators [32], (3.2) says that $\operatorname{Ran} E_{\left(-\infty, \Sigma_{3}\right)}(H) \Omega^{+}=\operatorname{Ran} E_{\left(-\infty, \Sigma_{3}\right)}(H) \Omega^{-}$and yields unitary of the $S$-matrix in the energy region $\left(\Sigma_{2}, \Sigma_{3}\right)$.

3. We will suppose that each $\operatorname{Ran} p_{\alpha}$ is finite dimensional. Since we are allowing $m=1$ and because of the possibility of the Effimov effect $[17,47]$, this can fail to be true. In that case, we just replace $\Sigma_{3}$ by $\Sigma_{3}-\varepsilon$ in the definition of $P_{\alpha}$ whence the new $P_{\alpha}$ 's are finite-dimensional and the proof below yields (3.2) with $E_{\left(-\infty, \Sigma_{3}\right)}$ replaced by $E_{\left(-\infty, \Sigma_{3}-\varepsilon\right)}$. Taking $\varepsilon$ to zero, (3.2) results.

The proof depends on 5 steps:

(1) Let $P$ be the projection onto the span of the $P_{\alpha}$ and let $Q=1-P$. We show that $Q E_{\left(-\infty, \Sigma_{3}-\varepsilon\right)}(H)$ is compact by using the basic equation (2.2). It will follow that $Q e^{-i t H} \tilde{E} \rightarrow 0$ strongly. 
(2) $P_{\alpha} P_{\beta}$ is compact. This is due to Combes [10].

(3) $P-\Sigma P_{\alpha}$ is compact. This will follow from step (2) and a general result appearing in Appendix A.

(4) $\Omega^{ \pm}\left(H_{\alpha}, H ; P_{\alpha}\right)$ exists. This will follow from the general Kato-Birman theory and the fact that $E_{I}\left(H_{\alpha}\right)\left(H_{\alpha} P_{\alpha}-P_{\alpha} H\right) E_{I}(H)$ is trace class for any bounded interval $I$.

(5) We will put it all together using ideas from [15].

\section{Step 1}

Lemma 3.2. $Q E_{\left(-\infty,\left(\Sigma_{3}-\varepsilon\right)\right)}(H)$ is compact for any $\varepsilon>0$.

Proof. It suffices to prove that $Q f(H)$ is compact for any continuous function $f$ with support in $\left(-\infty, \Sigma_{3}\right)$. By the basic equation (2.2) and its analysis in $\S 2$, this follows if we show that $Q f\left(H_{\alpha}\right)=0$. But $Q\left(1-P_{\alpha}\right)=Q$ since $P P_{\alpha}=P_{\alpha}$, and $\left(1-P_{\alpha}\right) f\left(H_{\alpha}\right)$ $=f\left(\left(1-P_{\alpha}\right) H_{\alpha}\right)=0$ since by definition of $P_{\alpha}, \sigma\left(H \uparrow\left(1-P_{\alpha}\right) \mathscr{H}\right) \subset\left[\Sigma_{3}, \infty\right)$.

In his paper, Combes proves the result that $Q H Q$ has purely discrete spectrum in $\left(-\infty, \Sigma_{3}\right)$. This is a strictly weaker than Lemma 3.2; see Appendix B.

Step 2. In terms of the tensor decomposition described in the definition of $P_{\alpha}$, we have $P_{\alpha}=p_{\alpha} \otimes_{\alpha} 1$. Explicitly, let $x_{\alpha}$ be a choice of internal coordinates for the cluster $C_{\alpha}^{(i)}$ and let $y_{\alpha}$ be the difference of the centers of mass. Then this tensor product is described by: $\varphi \otimes_{\alpha} \psi=\varphi\left(x_{\alpha}\right) \psi\left(y_{\alpha}\right)$. In [10], Combes proved a general result that if $A=a_{\alpha} \otimes_{\alpha} 1$ and $B=b_{\beta} \otimes_{\beta} 1$ and if $\alpha \neq \beta, a_{\alpha}$ and $b_{\beta}$ are Hilbert-Schmidt, then $A B$ is Hilbert-Schmidt. One can avoid the proof of this general result by using some special properties of the system under consideration:

Lemma 3.3. If $\alpha \neq \beta$, then $P_{\alpha} P_{\beta}$ is compact.

Proof. Let $y_{\alpha}$ be the center of mass coordinate for $\alpha$ and let $q_{\alpha}=\hbar / i \partial / \partial y_{\alpha}$ be the conjugate momentum. Then $y_{\alpha}$ and $q_{\alpha}$ commute with $P_{\alpha}$ so:

$$
P_{\alpha} P_{\beta}=\left[P_{\alpha}\left(1+y_{\alpha}^{2}\right)^{-1}\left(1+q_{\alpha}^{2}\right)^{-1}\right]\left[P_{\alpha}\left(1+y_{\alpha}^{2}\right)\left(1+q_{\alpha}^{2}\right) P_{\beta}\right]
$$

The first factor is compact since $\left(1+y_{\alpha}^{2}\right)^{-1}\left(1+q_{\alpha}^{2}\right)^{-1}=1 \otimes_{\alpha} c$ with $c$ compact, so we need only show that the second factor is bounded. Now since $\alpha \neq \beta, y_{\alpha}=x_{\alpha}+x_{\beta}$, for suitable coordinates, $x_{\alpha}$ and $x_{\beta}$ internal to $\alpha$ and $\beta$. Similarly $q_{\alpha}=l_{\alpha}+l_{\beta}$ for internal. Thus we only need show that $P_{\alpha} l_{\alpha}^{i} x_{\alpha}^{j} \varphi \in L^{2}$ for any discrete eigenfunction $\varphi$ of $h_{\alpha}$. That this is so is a consequence of a general theorem on exponential falloff of bound state wavefunctions $[28,12]$. (Actually all these authors prove that $\varphi$ falls exponentially. But then, so does $H \varphi$ and so $H_{0} \varphi$. It follows that $|l|^{2} \hat{\varphi}$ are $\hat{\varphi}$ are analytic in tubes with $L^{2}$ cross-sections so that $l_{\alpha}^{i} \hat{\varphi}$ has this property; (see [40] for arguments of this genre.)

Step 3 is discussed in Appendix A.

Step 4

Lemma 3.4. $E_{I}\left(H_{\alpha}\right) P_{\alpha} I_{\alpha} E_{I}(H)$ is trace class.

Proof. The proof is similar to that of Lemma 3.3. First note that we need only consider a single $V_{i j}$ term in $I_{\alpha}$ and a case where $p_{\alpha}$ is one dimensional since the 
general case is a finite sum of these cases. Then $P_{\alpha}$ reduces $H_{\alpha}$ and $P_{\alpha} H_{\alpha}=P_{\alpha}\left(e+T_{\alpha}\right)$ $=\left(e+T_{\alpha}\right) P_{\alpha}$ for suitable $e$ so that $E_{I}\left(H_{\alpha}\right) P_{\alpha}\left(I+T_{\alpha}\right)^{1 / 2 m+\varepsilon} \equiv A$ is bounded. Thus:

$$
\begin{aligned}
& E_{I}\left(H_{\alpha}\right) P_{\alpha} V_{i j} E_{I}(H)=A\left[\left(1+T_{\alpha}\right)^{-1 / 2 m-\varepsilon}\left(1+y_{\alpha}^{2}\right)^{-1 / 2 m-\varepsilon} P_{\alpha}\right] \\
& {\left[P_{\alpha}\left(1+y_{\alpha}^{2}\right)^{m / 2+\varepsilon} V_{i j}\left(H_{0}+1\right)^{-1}\right]\left[\left(H_{0}+1\right) E_{I}(H)\right] .}
\end{aligned}
$$

By (3.1), $D(H)=D\left(H_{0}\right)$, so the last factor is bounded. The second factor is clearly trace class, so we only need show that the third factor is bounded. But $y_{\alpha}=c\left(x_{i}-x_{j}\right)+x_{\alpha}$ so this is bounded as in Lemma 3.3.

\section{Step 5}

Proof of Theorem 3.1. By Pearson's form [29] of Birman's theorem and Lemma 3.4, the operators

$$
W_{\alpha}^{ \pm}=s-\lim _{t \rightarrow \mp \infty} e^{i t H_{\alpha}} P_{\alpha} e^{-i t H} E_{\mathrm{ac}}(H)
$$

exist, since $H_{\alpha} P_{\alpha}-P_{\alpha} H=P_{\alpha}\left(H_{\alpha}-H\right)=-P_{\alpha} I_{\alpha}$. Let $\varphi \in \operatorname{Ran} E_{\mathrm{ac}}(H) E_{\left(-\infty, \Sigma_{3}-\varepsilon\right)}(H)$. Then by Lemma 3.1, $Q e^{-i t H} \varphi \rightarrow 0$ as $t \rightarrow \pm \infty$ so $Q e^{-i t H} \tilde{E} \rightarrow 0$ strongly. By Lemma 3.3 and Theorem A.1 (in the first Appendix) $P-\sum_{\alpha} P_{\alpha}$ is compact, so $\left(P-\sum P_{\alpha}\right) e^{-i t H} \tilde{E} \rightarrow 0$ strongly.

Thus for $\varphi \subset \operatorname{Ran} \tilde{E}$ and $t \rightarrow \mp \infty$ :

$$
\begin{aligned}
e^{-i t H} \varphi & =\sum_{\alpha} P_{\alpha} e^{-i t H} \varphi+0(t) \\
& =\sum_{\alpha} e^{-i t H_{\alpha}} P_{\alpha} W_{\alpha}^{ \pm} \varphi+0(t)
\end{aligned}
$$

for since $W_{\alpha}^{ \pm}$exist and $e^{-i t H_{\alpha}}$ is unitary,

$$
\left[P_{\alpha} e^{-i t H_{\alpha}} W_{\alpha}^{ \pm} \varphi-P_{\alpha} e^{-i t H} \varphi\right] \rightarrow 0 \quad \text { as } \quad t \rightarrow \mp \infty
$$

so that

$$
\varphi=\sum_{\alpha} \hat{\Omega}_{\alpha}^{ \pm} W_{\alpha}^{ \pm} \varphi
$$

This shows that $\varphi \in \oplus \operatorname{Ran} \hat{\Omega}_{\alpha}^{ \pm}$.

We remark that (3.1) only need hold for the $V$ 's in an $I_{\alpha}$ with $P_{\alpha} \neq 0$. In particular, there could be Coulomb forces inside a cluster. Even if some long range forces are present below $\Sigma_{3}$, one can prove completeness below the lowest energy where long range force occur. With these methods, it should be possible to discuss scattering of an electron off a neutral atom below $\Sigma_{3}$.

\section{§4. On the Infinitude or Finiteness of the Number of Bound States of an $\mathbf{N}$-Body Quantum System II.}

This section should be viewed as a sequel to a paper of mine [38] with the same title, Part I. In that paper, various conjectures were made which are proven here. Hunziker [23] obtained a partial result related to those conjectures. Moreover, Combes [11] described a method to the author several years ago which he said 
would also prove these conjectures : there is some overlap between our methods and Combes. There is also some overlap with ideas of Thirring [45]. The basic result is :

Theorem 4.1. For each $\alpha$, let $P_{\alpha}$ be a reducing subspace for $H_{\alpha}(\{0\}$ is not a priori forbidden) and let

$$
\begin{aligned}
& \Sigma_{\alpha}^{\prime}=\inf \sigma\left(H_{\alpha} \uparrow\left(1-P_{\alpha}\right) \mathscr{H}\right) \\
& \Sigma_{\alpha}=\inf \sigma\left(H_{\alpha}\right) .
\end{aligned}
$$

Let $\Sigma=\min _{\alpha} \Sigma_{\alpha}$ and $\Sigma^{\prime}=\min _{\alpha} \Sigma_{\alpha}^{\prime}$ and suppose that $0 \geqq \Sigma^{\prime}>\Sigma$. Let $P$ be the projection onto the span of the $P_{\alpha}$ and let $Q=1-P$. Then for any $\delta<\left(\Sigma^{\prime}-\Sigma\right)$ there is an $n_{\delta}(<\infty)$ with

$$
\operatorname{dim} E_{(-\infty, a)}(H) \leqq \operatorname{dim} E_{(-\infty, a)}\left(\tilde{H}_{\delta}\right)+n_{\delta}
$$

for all $a \leqq \Sigma$ where

$$
\tilde{H}_{\delta}=P H P-\delta^{-1}(P H Q H P) .
$$

Proof. As in the proof of Lemma 3.2, $Q E_{\left(-\infty, \Sigma^{\prime}-\varepsilon\right)}$ is compact for any $\varepsilon>0$. It follows from general principles (see Theorem B.1), that $\sigma_{\text {ess }}(Q H Q) \subset\left(\Sigma^{\prime}, \infty\right)$. In particular,

$$
\operatorname{dim} E_{(-\infty, \Sigma)}(Q(H-\delta) Q) \equiv n_{\delta}<\infty .
$$

Now, by the operator inequality

$$
A^{*} B+B^{*} A \leqq A^{*} A+B^{*} B
$$

with $A=\delta^{-1 / 2} Q H P$ and $B=-Q \delta^{1 / 2}$

$$
P H Q+Q H P \geqq-\delta^{-1} P H Q H P-\delta Q^{2}
$$

so that

$$
H \geqq Q(H-\delta) Q+P\left[P H P-\delta^{-1} P H Q H P\right] P
$$

(4.1) now follows from (4.2) and the orthogonality of $P$ and $Q$.

Further developements now depend on the form of $P$. The simplest case occurs when, there is exactly one $\alpha$ with $\Sigma_{\alpha}=\Sigma$ and when the corresponding $h_{\alpha}$ has a simple discrete eigenvalue at the bottom of its spectrum. We will call this case where the bottom of the continuum is simple two body. This will be the generic situation for strong coupling in systems without any symmetry among particles. In this case, $P_{\alpha}$ is one dimensional $h_{\alpha} p_{\alpha}=p_{\alpha} \Sigma$ and $P=P_{\alpha}$. Thus $\operatorname{Ran} P_{\alpha} \cong L^{2}\left(\mathbb{R}^{m}\right)$ and, since $P_{\alpha} H Q_{\alpha}=P_{\alpha}\left(H-H_{\alpha}\right) Q_{\alpha}$ :

$$
\tilde{H}_{\delta} \cong-(2 m)^{-1} \Delta_{y}+\Sigma+W_{1}-\delta^{-1} W_{2}+\delta^{-1} W_{1}^{2}
$$

where $m^{-1}=\left(\sum_{i \in C_{\alpha^{\prime}}^{(1)}} \mu_{i}\right)^{-1}+\left(\sum_{i \in C_{\alpha}^{(2)}} \mu_{i}\right)^{-1}$ with $\mu_{i}=$ mass of particle $i$,

$$
\begin{aligned}
& W_{1}(y)=\int I_{\alpha}\left(y, x_{\alpha}\right)\left|\eta\left(x_{\alpha}\right)\right|^{2} \\
& W_{2}(y)=\int I_{\alpha}\left(y, x_{\alpha}\right)^{2}\left|\eta\left(x_{\alpha}\right)\right|^{2}
\end{aligned}
$$

where $\eta$ is the eigenfunction of $h_{\alpha}$.

From this computation follows a number of explicit results. For example: 
Theorem 4.2. Let $m \geqq 3$ and suppose that $V_{i j} \in L^{2} \cap L^{3 / 2}(m=3)$ or $L^{m / 2}(m>3)$ for each $i, j$ in different clusters for some breakup $\alpha$. If $m \geqq 3$, suppose moreover that $\hat{V}_{i j} \in L^{1}+L^{p}, p<m / m-2$ all $i, j$. Suppose that the bottom of the continuum is simple two body corresponding to the two body breakup $\alpha$. Then $H$ has finite spectrum below $\Sigma$.

Proof. We begin by recalling two facts: under the hypothesis on $\hat{V}_{i j}$, the Fourier transform of $V_{i j}$, the eigenfunction $\eta$ obeys

$$
\left|\eta\left(x_{\alpha}\right)\right| \leqq C \exp \left(-D\left|x_{\alpha}\right|\right)
$$

for some $C, D>0$ (see, e.g. [40]). Secondly, if $W \in L^{m / 2}\left(\mathbb{R}^{m}\right), m \geqq 3$, then $-\Delta+W$ has only finitely many negative eigenvalues (this follows from the Birman-Schwinger principle $[6,35]$ and a Sobolev estimate).

By the estimate (4.6) any partial integral of $\left|\eta\left(x_{\alpha}\right)\right|^{2}$ is in $L^{1} \cap L^{\infty}$, so by Young's inequality and the fact that $\int V_{i j}\left(x_{i}-x_{j}\right)\left|\eta\left(x_{\alpha}\right)\right|^{2} d x_{\alpha}$ is a convolution of $V_{i j}$ and a partial integral of $|\eta|^{2}$, the functions $W_{1}$ and $W_{2}$ lie in $L^{m / 2}\left(\mathbb{R}^{m}\right)$. Thus $-(2 m)^{-1} \Delta$ $+W_{1}-\delta^{-1} W_{2}$ has finitely many negative eigenvalues.

Remarks. 1. Yafeev [48] has proven related results. In a recent paper, Sigal [36] has proven that many $N$-body systems with short range forces have only finitely many bound states. He does not need to assume that the bottom of the continuum is simple two body but he needs to assume a condition [called condition (A)] which, while it holds for generic coupling constants, does not seen especially easy to check in any situation such as that envisaged above. For three body systems, our result is implied by results of Combescure-Moulin and Ginibre [13,19].

2. The most serious defect in the above result is that it is nonexplicit, i.e. while it does show $\operatorname{dim} E_{(-\infty, \Sigma)}$ is finite, it does not estimate it. Using available bounds (reviewed, e.g. in [41], see Remark 3 below), it is easy to estimate $\operatorname{dim} E_{(-\infty, 0)}$ $(-\Delta+W)$ and thus the contribution of $P H P-\delta^{-1} P H Q H P$. Unfortunately, the proof that $\operatorname{dim} E_{(-\infty, E)}(Q(H-\delta) Q)$ is finite is sufficiently indirect that it seems difficult to estimate it.

3. Not only is $\operatorname{dim} E_{(-\infty, 0)}(-\Delta+W)<\infty$ if $W \in L^{m / 2}(m \geqq 3)$; it is bounded by a multiple of $\int|W(x)|^{m / 2} d x$ by recent deep results $[14,27,34]$.

4. One can also prove results in dimensions $m=1$ and 2 but not with only $L^{p}$ hypotheses on $V$. For example using the bound [7]

$$
\operatorname{dim} E_{(-\infty, 0)}\left(-\frac{d^{2}}{d x^{2}}+V(x)\right) \leqq 1+\int|x||V(x)| d x
$$

and (4.3), one can prove an $m=1$ case of Theorem 4.2 if one supposes

$$
\int|x|\left|V_{i j}(x)\right| d x<\infty \text {. }
$$

5. One can easily weaken the condition on the $V_{i j}$ at infinite very slightly, e.g. if $V_{i j}$ can be written as an $L^{m / 2}$ function plus a weak $-L^{m / 2}$ function of arbitrarily small norm, the result remains true.

In cases where the bottom of the continuum is simple two body, Theorem 4.1 is able to handle quite sensitive situations. For example, consider the following model 


$$
\text { in } \begin{aligned}
L^{2}\left(\mathbb{R}^{3 N-3}\right) \\
\qquad \begin{aligned}
H= & -\sum_{i=1}^{N}(2 m)^{-1} \Delta_{i}-(2 \mu)^{-1} \Delta_{N+1}-\sum_{i=1}^{N+1} N\left|x_{1}\right|^{-1} \\
& +\sum_{1 \leqq i<j \leqq N+1}\left|x_{i}-x_{j}\right|^{-1}
\end{aligned}
\end{aligned}
$$

with $\mu<m$. This is almost the Hamiltonian of a positive ion, but we have artifically made one "electron" light to assure that the botton of the continuum is simple two body. We ignore statistics among the remaining electrons and take infinite nuclear mass, but see the remarks following the following theorem.

Theorem 4.3. The Hamiltonian $H$ of (4.7) has finitely many bound states below the continuum limit, $\Sigma$.

Proof. It suffices to prove that $W_{1}$ and $W_{2}$ given by $(4.1-2)$ are in $L^{3 / 2}\left(R^{3}\right)$. Since the ground state $\eta$ is spherically and permutation symmetric, we have:

$$
W_{1}(y)=-N|y|^{-1}+N \int\left|\eta\left(x_{\alpha}\right)\right|^{2}\left[\max \left(\left|x_{1}\right|,|y|\right)\right]^{-1} d^{3 N} x_{i}
$$

which is $O\left(e^{-c|y|}\right)$ by (4.3) and so in $L^{3 / 2}$. To control the $W_{2}$ term, write $|x|^{-1}=A(x)+B(x)$ with $A$ supported in $|x| \leqq 1$ and $B$ in $|x| \geqq 1$. If we can show that the $A^{2}$ and $B^{2}$ contributions to $W_{2}$ are in $L^{3 / 2}$, then $W_{2}$ is in $L^{3 / 2}$ since the $A B$ terms can be controlled by the Schwarz inequality. The $A^{2}$ term can be shown to give a contribution in $L^{3 / 2}$ as in the proof of Theorem 4.2. Thus we are left with the term $W_{2}^{\prime}$ given by (4.5) with

$$
I_{\alpha}^{\prime}=-N B\left(x_{N+1}\right)+\sum_{i=1}^{N} B\left(x_{i}-x_{N+1}\right) .
$$

Now, the bound

$$
|B(x-y)-B(y)| \leqq(|y|+1)^{-2}\left[16|x|+2(2(x)+1)^{2}+18\right]
$$

holds since the $16|x|$ term controls the case $|y| \geqq 2|x|,|y| \geqq 2$, the $2(|x|+1)^{2}$ term the case $2|x| \geqq|y|$ and the 18 term the region $|y| \leqq 2$. Thus, by (4.3), $\left|W_{2}^{\prime}(y)\right| \leqq C(|y|+1)^{-4}$ is in $L^{3 / 2}$.

Remarks. 1. A finite nuclear mass is trivially accomadated in the above. With two additional remarks, one can accomadate the case where one imposes Fermi statistics on the electrons of mass $m$. First the eigenvalue of $h_{\alpha}$ may be degenerate which requires some small modifications. Secondly, $\eta$ may not be spherically symmetric but by parity invariance, the total charge distributor of the "electrons" and nucleus seen by the "test" charge $N+1$ will be one without any dipole moment so that $W_{1}(y)$ will certainly be at worst $0\left(y^{-3}\right)$.

2. This theorem will not remain true if the single nuclear charge $N|x|^{-1}$ is replaced by $\sum_{i=1}^{m} z_{i}\left|x-x_{i}\right|^{-1}$ with $\sum_{i=1}^{m} z_{i}=N, z_{i}>0$. For, the ground state of the $H_{\alpha}$ system will have a charge distribution which when combined with the nuclear charge distribution has no net charge but in general a non-zero dipole moment. If this dipole moment is large, there results on $r^{-2}$ potential with an infinity of bound states. Lieb (private communication) has remarked that one can get such a moment if $N=1, Z_{1}=1 / 3, Z_{2}=2 / 3,\left|x_{1}-x_{2}\right|$ large. The electron will "stick" to the second charge. 
3. For other results on bound states of positive ionic Coulomb systems see $[1,2$, $48,52,53,54]$.

Finally, we consider how quickly $\operatorname{dim} E_{(-\infty, a)}$ grows as $a \rightarrow \Sigma$ in cases where $\operatorname{dim} E_{(-\infty, \Sigma)}=\infty$. We rely on results on the analogous two-body problem due to Brownell-Clark [8] and McLeod [55] in the following form:

Lemma 4.4. Let $m \geqq 3$ and let $V=V_{1}+V_{2}$ where $V_{1} \in L^{m / 2}\left(\mathbb{R}^{m}\right)$ and $V_{2}$ is bounded and obeys as $x \rightarrow \infty$

$$
V_{2}(x)=-C x^{-\beta}+\sum_{i=1}^{k} d_{i} x^{-\beta-q_{i}}+0\left(x^{-2-\varepsilon}\right)
$$

for some $\beta \in(0,2)$ and some $C>0$ and $q_{i}>0$. Let

$$
N(\alpha)=\operatorname{dim} E_{(-\infty,-\alpha)}\left(-(2 \mu)^{-1} \Delta+V\right)
$$

and let

$$
\begin{aligned}
g(\alpha) & =D \alpha^{-v m} \\
v & =\beta^{-1}-\frac{1}{2} \\
D & =(2 \pi)^{-m} \tau_{m}(2 \mu)^{m / 2} C^{m / 2}\left[\int_{0}^{1}\left(1-y^{\beta}\right)^{m / 2} y^{-\beta m / 2} y^{m-1} d y\right]
\end{aligned}
$$

with $\tau_{m}$ the volume of the unit ball in $\mathbb{R}^{m}$. Then

$\lim _{\alpha \downarrow 0} N(\alpha) / g(\alpha)=1$.

Proof. Follows from Theorem XIII.82 of [33] as extended in Problem 132 of Chapter XIII of [33].

Remark. The integral in (4.9c) is doable in terms of $\Gamma$ functions. The function $g(\alpha)$ is just the volume in $\mathbb{R}^{2 m}$ of the region of phase space where $(2 \mu)^{-1} p^{2}-C x^{-\beta}<-\alpha$; Lemma 4.4 is just an expression that the small $\alpha$ region is quasiclassical.

Theorem 4.5. Let $H$ be the Hamiltonian of an $N$ body system of $m$-dimensional particles, $m \geqq 3$. Suppose that the bottom of the continuum is simple two body. Let $\alpha=\left(C_{\alpha}^{(1)}, C_{\alpha}^{(2)}\right)$ for the cluster determining $\Sigma$. Suppose that, for each $i \in C_{\alpha}^{(1)}, j \in C_{\alpha}^{(2)}$, $V_{i j}=V_{i j}^{(1)}+V_{i j}^{(2)}$ with $V_{i j}^{(1)}$ obeying the hypothesis of Theorem 4.2 and with $V^{(2)}$ obeying (4.8) with the following provisos: (a) $\beta$ is independent of $i, j$ and is in $(0,2)$. (b) $C_{i j}$ need not be positive or even non-zero but $C \equiv \sum C_{i j}>0$. (c) $q$ may be $i, j$ dependent. (d) The $0\left(x^{-2-\varepsilon}\right)$ term has a Fourier transform in $L^{1}+L^{p}$ with $p<m / m-2$. Then

$$
g(\alpha)^{-1} \operatorname{dim} E_{(-\infty, \Sigma-\alpha)}(H) \rightarrow 1
$$

as $\alpha \downarrow 0$, where $\mu$ is the reduced mass of the clusters in $\alpha$.

Proof. By Theorem 4.1 and the elementary variational principle result [28] $\operatorname{dim} E_{(-\infty, a)}(P H P) \leqq \operatorname{dim} E_{(-\infty, a)}(H)$ for $a<0$, we need only prove that

$$
\begin{aligned}
& g(\alpha)^{-1} \operatorname{dim} E_{(-\infty, \alpha)}\left(-(2 \mu)^{-1} \Delta+W_{1}\right) \rightarrow 1 \\
& g(\alpha)^{-1} \operatorname{dim} E_{(-\infty,-\alpha)}\left(-(2 \mu)^{-1} \Delta+W_{1}-\delta^{-1} W_{2}\right) \rightarrow 1 .
\end{aligned}
$$

This follows from Lemma 4.4 and the type of estimates developed so far.

This result is not comparable to that of Hunziker [23] who obtains information on a limited fraction of the eigenvalues rather than just dimensions.

We end this section by noting that it should be easy to extend our method to the case where a single two-body cluster decomposition determines the bottom of the 
spectrum but the eigenvalues are not simple - this can happen only when $H$ is restricted to some symmetry subspace. It should also be possible to discuss $\mathrm{H}^{-}$with infinite nuclear mass since the $P_{\alpha}$ 's in that case commute. The interesting open problem concerns non-orthogonal $P_{\alpha}$ 's.

\section{Appendix A. Almost Mutually Orthogonal Projections}

Theorem A.1. Let $\left\{P_{\alpha}\right\}_{\alpha=1}^{N}$ be a family of orthogonal projections on a Hilbert space and $\mathscr{H}$ and let $P$ be the projection onto the span of the $\operatorname{Ran} P_{\alpha}$. Suppose that $P_{\alpha} P_{\beta}$ is compact for each $\alpha \neq \beta$. Then $P-\sum_{\alpha=1}^{N} P_{\alpha}$ is compact.

Remark. R. Kadison and P. Deift (private communications) have remarked independently that this theorem has a true converse essentially by the following argument: $P_{1}+P_{2} \leqq\left(\Sigma P_{\alpha}-P\right)+P$. Thus since $P_{1} P P_{1}=P_{1}$ we have that $P_{1} P_{2} P_{1} \leqq P_{1}\left(\Sigma P_{\alpha}-P\right) P_{1}$. Thus, if $\left(\Sigma P_{\alpha}-P\right)$ is compact, so is $P_{1} P_{2} P_{1}$ since it is positive. As a result $\left|P_{2} P_{1}\right|=\left(P_{1} P_{2} P_{1}\right)^{1 / 2}$ is compact, and thus, so is $P_{2} P_{1}$. Deift has also given a slightly more complicated proof showing that if $P-\Sigma P_{\alpha} \in \mathscr{I}_{p}$, then each $P_{\alpha} P_{\beta}$ is in $\mathscr{I}_{p}$ also

Proof. Suppose first that we have the theorem for $N=2$ and for $N=n-1$. Given $n$ projections, let $P_{1}^{\prime}$ be the projection on the span of $P_{1}, \ldots, P_{n-1}$ and let $P_{2}^{\prime}=P_{n}$. By the theorem for $N=n-1, P_{1}^{\prime}-P_{1} \ldots P_{n-1}$ is compact so that $P_{1}^{\prime} P_{2}^{\prime}$ is compact. Thus by the result for $N=2, P-P_{1}^{\prime}-P_{2}^{\prime}$ is compact proving the result for $N=n$. It follows by induction that we need only consider the case $N=2$.

Suppose that $N=2$. Since $P_{1} P_{2} P_{1}$ is compact, we can find an orthonormal basis for Ran $P_{1}$ of eigenfunctions for $P_{1} P_{2} P_{1}$. Organize these in three sets $\left\{\varphi_{i}\right\}_{i=1}^{N}(N$ may be infinite), $\left\{\varphi_{i}^{\prime}\right\}_{i=1}^{N^{\prime}}\left(N^{\prime}<\infty\right),\left\{\varphi_{i}^{\prime \prime}\right\}_{i=1}^{N^{\prime \prime}}\left(N^{\prime \prime}\right.$ may be infinite), so that $\left(P_{1} P_{2} P_{1}\right) \varphi_{i}=\alpha_{i} \varphi_{i}\left(0<\alpha_{i}<1\right), \quad\left(P_{1} P_{2} P_{1}\right) \varphi_{i}^{\prime}=\varphi_{i}^{\prime}, \quad$ i.e. $\quad \varphi_{i}^{\prime} \in \operatorname{Ran} P_{1} \cap \operatorname{Ran} P_{2} \quad$ and $P_{1} P_{2} P_{1} \varphi_{i}^{\prime \prime}=0$, i.e. $\varphi_{i}^{\prime \prime} \in\left(\operatorname{Ran} P_{1}\right) \cap\left(\operatorname{Ran} P_{2}\right)^{\perp}$. Similarly pick a basis $\left\{\psi_{i}\right\} \cup\left\{\psi_{i}^{\prime}\right\} \cup\left\{\psi_{i}^{\prime \prime}\right\}$ for $\operatorname{Ran} P_{2}$ with $\psi_{i}^{\prime}=\varphi_{i}^{\prime}$ and $\psi_{i}=\alpha_{i}^{-1 / 2} P_{2} \varphi_{i}$; it is easy to check that these $\psi$ 's are orthonormal. Now let

$$
\begin{aligned}
& \eta_{i}=n_{i}\left(\varphi_{i}+\psi_{i}\right) \\
& \gamma_{i}=n_{i}^{\prime}\left(\varphi_{i}-\psi_{i}\right)
\end{aligned}
$$

where $n_{i}^{-2}=2+2 \alpha_{i}^{1 / 2},\left(n_{i}^{\prime}\right)^{-2}=2-2 \alpha_{i}^{1 / 2}$. Then $\left\{\varphi_{i}^{\prime}\right\}_{i=1}^{N^{\prime}} \cup\left\{\varphi_{i}^{\prime \prime}\right\} \cup\left\{\psi_{i}^{\prime \prime}\right\} \cup\left\{\eta_{i}\right\} \cup\left\{\gamma_{i}\right\}$ is a basis for $\operatorname{Ran} P$. On these 5 sets, $P-P_{1}-P_{2}$ has eigenvalues, $1,0,0,-\alpha_{i}^{1 / 2}, \alpha_{i}^{1 / 2}$. Since $N^{\prime}<\infty$ and $\alpha_{i} \rightarrow 0, P-P_{1}-P_{2}$ is compact.

Remark. 1. Notice, that $\alpha_{i}^{1 / 2}$ are the eigenvalues of $\left(P_{1} P_{2} P_{1}\right)^{1 / 2}=\left|P_{1} P_{2}\right|$, so we have that $P-\Sigma P_{\alpha}$ is in some trace ideal, $\mathscr{I}_{p},[16,21,42]$ if each $P_{\alpha} P_{\beta}$ is in that trace ideal.

2. Two different proofs of this result have been provided by $\mathrm{P}$. Deift and $\mathrm{R}$. Kadison.

\section{Appendix B. Compactness of $Q E(H)$ and $\sigma_{\text {ess }} Q H Q$}

Let $H$ be an arbitrary self-adjoint operator which is bounded from below. For any arbitrary orthogonal projection $Q$, one can define a "self-adjoint" operator $Q H Q$; we say "self-adjoint", since it may not have a dense domain-such objects have been 
considered recently $[26,43,44]$. Let $a \leqq 0$. We want to consider the pair of statements :

(1) $Q E_{(-\infty, a-\varepsilon)}(H)$ is compact for each $\varepsilon>0$.

(2) $\sigma_{\text {ess }}(Q H Q) \subset[a, \infty)$.

For Schrödinger operators, $H$, results of type (2) come from resolvent equation methods [9]; results of type (1) are proven in the text. Here we want to show that on an abstract level (1) implies (2) but not conversely.

Theorem B.1. Under the above considerations, (1) implies (2).

Proof. Fix $\varepsilon>0$. Write

$$
Q H Q=Q H E_{(-\infty, a-\varepsilon)}(H) Q+Q H E_{[a-\varepsilon, \infty)}(H) Q \equiv A_{\varepsilon}+B_{\varepsilon} .
$$

Then $B_{\varepsilon} \geqq a-\varepsilon$ while $A_{\varepsilon}$ is compact if (1) holds. It follows that $\sigma_{\text {ess }}\left(A_{\varepsilon}+B_{\varepsilon}\right) \subset[a-\varepsilon, \infty)$ for any $\varepsilon>0$ on account of Weyl's theorem (see Section XIII.4 of [33]).

Example. Let $\mathscr{H}=l_{2}(0, \infty)$, i.e. sequences $\left(a_{1}, a_{2}, \ldots\right)$. Let $(H a)_{n}=\left[1+2(-1)^{n}\right] a_{n}$; i.e. $H\left(a_{1}, a_{2}, \ldots\right)=\left(-a_{1}, 3 a_{2},-a_{3}, \ldots\right)$. Let $Q$ be the projection onto vectors with $a_{2}=a_{1}$, $a_{4}=a_{3}$, etc. Then, for $0<\varepsilon<1$,

$$
Q H Q=Q, Q E_{(-\infty,-\varepsilon)}(H) Q=-\frac{1}{2} Q
$$

so (2) is true for $a=0$, but (1) is not.

\section{References}

1. Antonec,M. A., Skereshevsky,I. A., Zhislin,G.M.: Teor. Mat. Fiz. 16, 235 (1973); Theoret. Math. Phys. 16, 800 (1974)

2. Antonec, M.A., Skereshevsky,I.A., Zhislin,G.M.: Dokl. Akad. Nauk. SSR 222; Soviet Math. Dokl. 18, 688 (1975)

3. Avron,J., Herbst,I., Simon, B.: Schrödinger operators with magnetic fields (in prep.)

4. Balslev, E.: Ann. Phys. 73, 49 (1972)

5. Balslev,E., Combes,J.M.: Commun. math. Phys. 22, 280 (1971)

6. Birman,M.S.: Math. Sb. 55, 124-174 (1961); AMS Transl. 53, 23-80 (1966)

7. Blankenbecler, R., Goldberger,M.L., Simon,B.: Ann. Phys., to appear

8. Brownell,F.H., Clark,C.W.: J. Math. Meth. 10, 31-70 (1961)

9. Combes, J.M.: Nuovo Cimento 64A, 111 (1969)

10. Combes,J.M.: Properties of some connected kernels in multiparticle Systems (1969) (Manuscript)

11. Combes,J.M. : Letter to the author, Fall (1972)

12. Combes,J.M., Thomas,L.: Commun. math. Phys. 34, 251 (1973)

13. Combescure,J., Ginibre,J.: Preprint (1975)

14. Cwickel,M.: Ann. Math. (to appear)

15. Deift,P., Simon,B.: Commun. Pure Appl. Math. (to appear)

16. Dunford,N., Schwartz,J.: Linear operators, Vol. II. New York: Interscience 1963

17. Effimov, V.N.: Phys. Lett. 33B, 563 (1970)

18. Enss, V.: Commun. math. Phys. 52, 233-238 (1977)

19. Ginibre,J., Moulin, M.: Ann. Inst. H.Poincaré 21, 97-145 (1974)

20. Glimm,J., Jaffe, A.: Ann. Math. 91, 362-401 (1970); see also J. Func. Anal. 7, 323-357 (1971)

21. Gohberg,I.C., Krein,M.: Introduction to the theory of non-self-adjoint operators. AMS Translations 1969 (Russian original 1965)

22. Hunziker,W.: Helv. Phys. Acta 39, 451 (1966)

23. Hunziker,W.: Helv. Phys. Acta 48, 145 (1975) 
24. Jörgens, K.: Tech. Rept. Univ. Heidelberg (unpublished) 1965

25. Jörgens, K., Weidmann, J. : Lecture notes in Mathematics, Vol. 313. Berlin-Heidelberg-New York: Springer (1973)

26. Kato,T.: Advan. Math. Krein anniv. issue (to appear)

27. Lieb,E.H.: Bull. AMS 82, 751-753 (1976); and in prep.

28. O'Connor, A.: Commun. math. Phys. 32, 319 (1973)

29. Pearson,D.: J. Func. Anal (to appear)

30. Reed,M., Simon, B.: Methods of modern mathematical physics, Vol. I. Functional analysis, New York-London: Academic Press 1972

31. Reed,M., Simon, B.: Methods of modern mathematical physics, Vol. II. Fourier analysis, selfadjointness. New York-London: Academic Press 1975

32. Reed,M., Simon, B.: Methods of modern mathematical physics, Vol. III. Scattering theory, New York-London: Academic Press 1978 (exp)

33. Reed, M., Simon, B. : Methods of modern mathematical physics, Vol. IV. Analysis of operators. New York-London: Academic Press 1977 (exp.)

34. Rosenbljum, G.: Dokl. Akad. Nauk SSR 202, 1012-1015 (1972); and in press

35. Schwinger,J.: Proc. Nat. Acad. Sci. 47, 122-129 (1961)

36. Sigal,I.: Commun. math. Phys. 48, 137 (1976)

37. Simon, B.: Quantum mechanics for Hamiltonians defined as quadratic forms. Princeton: Univ. Press 1971

38. Simon, B. : Helv. Phys. Acta 43, 607 (1970)

39. Simon,B.: Commun. math. Phys. 27, 1 (1972)

40. Simon, B.: Proc. Am. Math. Soc. 42, 395 (1974)

41. Simon,B.: Contribution to: Studies in mathematical physics, essays in honor of Valentine Bargmann, Princeton Univ. Press 1976

42. Simon, B.: Adv. Math. (to appear)

43. Simon, B.: J. Func. Anal. (to appear)

44. Simon,B.: Proc. Roy. Soc. Edinburgh, to appear

45. Thirring, W.: Vorlesungen über mathematische Physik. Univ. Wien Lecture Notes

46. van Winter, C.: Mat. Fys. Skr. Dan. Vid. Selsk. 2, Nos. 8, 10 (1964-65)

47. Yafeev,D. R.: Mat. Sb. 94, (1974); Math. USSR Sb. 23, 535 (1974)

48. Yafeev,D. R.: Func. Anal. Pril 6, 103 (1972); Func. Anal. Appl. 6, 349 (1972)

49. Zhislin, G.M.: Trud. Musk. Math. Obsc. 9, 82-120 (1960)

50. Zhislin, G.M. : Dokl. Akad. Nauk SSR 175, 521-524 (1967); Soviet Math. Dokl 8, 878-882 (1967)

51. Zhislin, G. M.: Izv. Akad. Nauk. SSR 33, 590-649 (1969); Math. USSR Izv. 3, 559-616 (1969/70)

52. Zhislin, G.M.: Dokl. Akad. Nauk SSR 207, 25 (1972); Soviet Math. Dokl. 13, 1445 (1972)

53. Zhislin, G.M.: Teor. Mat. Fiz. 21, 60 (1974); Theoret. Math. Phys. 21, 971 (1974)

54. Zhislin, G.M., Sigalovz, A.G.: Izv. Akad. Nauk 29, 835-860 (1965); AMS Transl. Ser. 2, 91, 297 (1970)

55. Uchiyama,J.: Publ. RIMS Kyto Univ. 5A, 51-63 (1969)

56. McLeod,J.: Proc. L.M.S. 11, 139-158 (1961)

Communicated by J. Ginibre

Received March 14, 1977

Notes added in proof.

1. Results similar to Lemma 2.4 occur in the Haag-Ruelle theory.

2. The considerations of $\S 2$ can be extended to handle non-self-adjoint $H$ 's such as occur in dilation analytic theory; this will be discussed in a forthcoming ETH Preprint by the author and others, to be submitted to Trans. Am. Math. Soc.

3. The considerations of $\S 3$ have been extended by the author to central potentials $0\left(|x|^{-1-\varepsilon}\right)$ at infinity and to electron scattering from neutral atoms; see a Weizmann Institute Preprint submitted to Commun. Math. Phys.

4. Zhislin has kindly informed the author that he and S. A. Vulgal'ter (Theo. Mat. Fiz. 32 (1977) No. 1) have found "the most general results of discrete spectrum finiteness... in the case where the bottom of the continuum is two body" including the results of $\S 4$. 